Optical properties of the twodimensional magnetoexcitons under the influence of the Rashba spin-orbit coupling

T. Hakioglu

M. A. Liberman

S. A. Moskalenko

I. V. Podlesny 


\title{
Optical properties of the two-dimensional magnetoexcitons under the influence of the Rashba spin-orbit coupling
}

\author{
T. Hakioglu ${ }^{\mathrm{a}}$, M.A. Liberman ${ }^{\mathrm{b}}$, S.A. Moskalenko ${ }^{\mathrm{cl}}$, and I.V. Podlesny ${ }^{\mathrm{c}}$ \\ ${ }^{a}$ Department of Physics, Bilkent University, 06800, Ankara, Turkey \\ ${ }^{\mathrm{b}}$ Department of Physics, Uppsala University, Box 530, SE-751 21, Uppsala, Sweden \\ ${ }^{c}$ Institute of Applied Physics, Academy of Sciences of Moldova, 5, Academiei str., MD-2028, \\ Chisinau, Republic of Moldova
}

\begin{abstract}
The influence of the Rashba spin-orbit coupling on the two-dimensional (2D) electrons and holes in a strong perpendicular magnetic field leads to different results of the Landau quantization in different spin projections. In Landau gauge the unidimensional wave vector describing the free motion in one in-plane direction is the same for both spin projections, whereas the numbers of the Landau quantization levels are different. For electron in $s$-type conduction band they differ by one, as was established earlier by Rashba ${ }^{1}$, whereas for heavy holes in $p$-type valence band influenced by the 2D symmetry of the layer they differ by three. There are two lowest spin-splitted Landau levels for electrons as well as two lowest for holes. They give rise to four lowest energy levels of the 2D magnetoexcitons. It is shown that two of them are dipole-active in band-to-band quantum transitions, one is quadrupole-active and the fourth is forbidden. The optical orientation under the influence of the circularly polarized light leads to optical alignment of the magnetoexcitons with different orbital momentum projections on the direction of the external magnetic field.
\end{abstract}

Keywords: magnetoexcitons, Rashba spin-orbit splitting, Landau quantization.

\section{INTRODUCTION}

The influence of the spin-orbit coupling (SOC) on the two-dimensional (2D) Wannier-Mott excitons in double quantum well (DQW) structures, as well as the possibilities of the nonconventional electron-hole (e-h) pairing in these conditions were discussed in Ref. ${ }^{2,3}$. The main results are the breaking of the spin degeneracy of the electrons and holes, the changes of the exciton structure, and new properties of the Bose-Einstein condensed excitons. There are two types of SOC. One of them described by Dresselhaus ${ }^{4}$ is known to be intrinsically present in zinc-blende structure. The Rashba spin-orbit coupling (RSOC) ${ }^{1,5}$ depends on the electric field strength $E_{z}$ perpendicular to the layer surface.

As was mentioned in Ref. ${ }^{6,7}$ the Rashba model can be described by purely group theoretical means. For electron in $s$-like conduction band the total angular momentum with spin-orbit interaction equals to $j=1 / 2$. Both wave vectors $\vec{k}$ and electric strength $\vec{E}$ are polar vectors, whereas their cross product $[\vec{k} \times \vec{E}]$ is an axial vector. Its point product with the spin axial vector $\vec{\sigma}$ gives rise to the triple scalar product $[\vec{k} \times \vec{E}] \cdot \vec{\sigma}$. This expression is an invariant under the action of the group symmetry elements forming identity representation $\Gamma_{1}$. In the first quantization representation the wave vector $\vec{k}$ is substituted by $-i \vec{\nabla}$. In the $\Gamma_{6}$-type conduction band the triple scalar product is the only term of the first order on $\vec{\nabla}$ and $\vec{E}$ compatible with the symmetry of the band.

The band structure described by the Hamiltonian with RSOC

1E-mail:exciton@phys.asm.md

ICONO 2010: International Conference on Coherent and Nonlinear Optics, edited by Claude Fabre,

Victor Zadkov, Konstantin Drabovich, Proc. of SPIE Vol. 7993, 79930N · C 2011 SPIE

CCC code: $0277-786 \mathrm{X} / 11 / \$ 18 \cdot$ doi: $10.1117 / 12.882290$ 


$$
H_{e}=-\frac{\hbar^{2} \Delta_{\|}}{2 m_{e}} \hat{I}-i \tilde{\alpha}\left(\hat{\sigma}_{x} \frac{\partial}{\partial y}-\hat{\sigma}_{y} \frac{\partial}{\partial x}\right) ; \Delta_{\|}=\frac{\partial^{2}}{\partial x^{2}}+\frac{\partial^{2}}{\partial y^{2}} ; \tilde{\alpha}=\alpha_{e} E_{z}
$$

has the dispersion laws

$$
E_{e}^{ \pm}=\frac{\hbar^{2} k_{\|}^{2}}{2 m_{e}} \pm|\tilde{\alpha}| k_{\|} ; k_{\|}=\sqrt{k_{x}^{2}+k_{y}^{2}}
$$

One of them contains the loop of minima ${ }^{1,5}$. The topmost valence band in our case is $p$-like with orbital quantum number $l=1$ and with the total angular momentum equal to $j=3 / 2$. The four-fold band states give rise to heavy and light holes forming in cubic crystals the irreducible representation $\Gamma_{8}$ in the point $k=0$.

For the LH the effective Rashba Hamiltonian has the lowest order in $\vec{k}$ term and is the same as for the conduction electrons. For the HH the effective Rashba Hamiltonian happens to be the third order in $\vec{k}$ and remains the first order in spin operators $\vec{\sigma}$ as follows ${ }^{6,7}$

$$
H_{h}^{S O C}=\beta_{h} E_{z}\left(\hat{\sigma}_{+} k_{-}^{3}+\hat{\sigma}_{-} k_{+}^{3}\right)
$$

where

$$
\begin{gathered}
\sigma_{ \pm}=\frac{1}{2}\left(\hat{\sigma}_{x} \pm i \hat{\sigma}_{y}\right) ; k_{ \pm}=\left(k_{x} \pm i k_{y}\right) ; \\
\hat{\sigma}_{x}=\left|\begin{array}{cc}
0 & 1 \\
1 & 0
\end{array}\right| ; \hat{\sigma}_{y}=\left|\begin{array}{cc}
0 & -i \\
i & 0
\end{array}\right| ; \hat{\sigma}_{z}=\left|\begin{array}{cc}
1 & 0 \\
0 & -1
\end{array}\right| ; \hat{I}=\left|\begin{array}{cc}
1 & 0 \\
0 & 1
\end{array}\right| .
\end{gathered}
$$

The electric field strength $E_{z}$ depends on the density of charges in the system ${ }^{6,7}$. The interaction constants were evaluated $\mathrm{in}^{2,3}$ for different values of $E_{z}$, arriving to the conclusion that at $E_{z}=100 \div 200 \frac{\mathrm{kV}}{\mathrm{cm}}$ the RSOC is a dominant mechanism for the energy band spin splitting.

\section{THE LANDAU QUANTIZATION OF 2D ELECTRONS AND HOLES IN THE PRESENCE OF RSOC}

Following the papers ${ }^{1-7}$ the full Hamiltonians describing the Landau quantization of the $2 \mathrm{D}$ electrons and holes in a strong perpendicular magnetic field taking into account the RSOC consist from two parts. First of them are the zero order Hamiltonians for electrons and holes in a strong perpendicular magnetic field

$$
H_{e}^{0}=\frac{\hbar^{2}}{2 m_{e}}\left(\hat{P}_{e}+\frac{|e|}{c} \vec{A}\left(\vec{r}_{e}\right)\right)^{2} ; H_{h}^{0}=\frac{\hbar^{2}}{2 m_{h}}\left(\hat{P}_{h}-\frac{|e|}{c} \vec{A}\left(\vec{r}_{h}\right)\right)^{2},
$$

where $\hat{P}_{e}$ and $\hat{P}_{h}$ are 2D momenta equal to $-i h \vec{\nabla}_{e}$ and $-i h \vec{\nabla}_{h}$ correspondingly and the vector potential $\vec{A}(\vec{r})$ is written in Landau gauge, i.e. $\vec{A}(\vec{r})=\left(A_{x}=-H y ; A_{y}=0 ; A_{z}=0\right)$. The second parts of the full Hamiltonians are the RSOC Hamiltonians (1) and (3), in which instead of usual momenta must be introduced the kinematic momenta

$$
\hat{P}_{e}+\frac{|e|}{c} \vec{A}\left(\vec{r}_{e}\right) \text { and } \hat{P}_{h}-\frac{|e|}{c} \vec{A}\left(\vec{r}_{h}\right),
$$

what is equivalent to write instead of $k_{x}^{e}, k_{y}^{e}, k_{x}^{h}$ and $k_{y}^{h}$ the new expressions

$$
\begin{gathered}
K_{x}^{e}=\left(-i \frac{\partial}{\partial x_{e}}-\frac{y_{e}}{l^{2}}\right) ; K_{y}^{e}=-i \frac{\partial}{\partial y_{e}} \\
K_{x}^{h}=\left(-i \frac{\partial}{\partial x_{h}}+\frac{y_{h}}{l^{2}}\right) ; K_{y}^{h}=-i \frac{\partial}{\partial y_{h}} ; l^{2}=\frac{\hbar c}{|e| H}
\end{gathered}
$$

correspondingly. $l$ is the magnetic length. The new operators $K_{x}$ and $K_{y}$ do not commute

$$
\left[K_{x}^{e}, K_{y}^{e}\right]=-\frac{i}{l^{2}} ;\left[K_{x}^{h}, K_{y}^{h}\right]=\frac{i}{l^{2}} \text {. }
$$


The RSOC Hamiltonians for 2D electrons and holes in a strong perpendicular magnetic field have the forms

$$
\begin{aligned}
& H_{e}^{S O C}=\alpha_{e} E_{z}\left[\hat{\sigma}_{x} K_{y}^{e}-\hat{\sigma}_{y} K_{x}^{e}\right] ; H_{h}^{S O C}=-\beta_{h} E_{z} \\
& \times\left\{\hat{\sigma}_{x}\left[K_{y}^{h^{3}}-\left(K_{x}^{h^{2}} K_{y}^{h}+K_{y}^{h} K_{x}^{h^{2}}+K_{x}^{h} K_{y}^{h} K_{x}^{h}\right)\right]\right. \\
& \left.+\hat{\sigma}_{y}\left[K_{x}^{h^{3}}-\left(K_{y}^{h^{2}} K_{x}^{h}+K_{x}^{h} K_{y}^{h^{2}}+K_{y}^{h} K_{x}^{h} K_{y}^{h}\right)\right]\right\} .
\end{aligned}
$$

The full Hamiltonians are

$$
H_{e}=H_{e}^{0}+H_{e}^{S O C} ; H_{h}=H_{h}^{0}+H_{h}^{S O C} .
$$

The solutions of the Landau quantization task for electrons and holes are chosen in the forms ${ }^{1,5}$

$$
\begin{gathered}
\left|\Psi_{e}(R, p ; x, y)\right\rangle=\frac{e^{i p x}}{\sqrt{L_{x}}}\left|\begin{array}{l}
\Phi_{1}(y, p) \\
\Phi_{2}(y, p)
\end{array}\right| ; \\
\left\langle\Psi_{e}(R, p ; x, y)\left|=\frac{e^{-i p x}}{\sqrt{L_{x}}}\right| \Phi_{1}^{*}(y, p) \quad \Phi_{2}^{*}(y, p)\right| ; \\
\left|\Psi_{h}(R, q ; x, y)\right\rangle=\frac{e^{i q x}}{\sqrt{L_{x}}}\left|\begin{array}{l}
f_{1}(y, q) \\
f_{2}(y, q)
\end{array}\right| ; \\
\left\langle\Psi_{h}(R, q ; x, y)\left|=\frac{e^{-i q x}}{\sqrt{L_{x}}}\right| \begin{array}{ll}
f_{1}^{*}(y, q) & f_{2}^{*}(y, q) \mid .
\end{array}\right.
\end{gathered}
$$

The actions of the operators $K_{x}^{e}$ on the function $e^{i p x_{e}}$ and of the operator $K_{x}^{h}$ on the function $e^{i q x_{h}}$ are

$$
K_{x}^{e} \exp \left(i p x_{e}\right)=\left(p-\frac{y_{e}}{l^{2}}\right) e^{i p x_{e}} ; K_{x}^{h} \exp \left(i q x_{h}\right)=\left(q+\frac{y_{h}}{l^{2}}\right) e^{i q x_{h}} .
$$

Instead of variables $y_{e}$ and $y_{h}$ we will introduce the dimensionless variables

$$
\eta_{e}=\frac{y_{e}}{l}-p l ; \eta_{h}=\frac{y_{h}}{l}+q l .
$$

The Schrödinger equations on the base of the full Hamiltonians (10) with the solutions in the forms (11) depend on two variables $x$ and $y$. But taking into account the translational symmetry in direction $x$ and the relations (12) these equations can be transformed in Schrödinger equations depending only on one variable. The cyclotron energies for electron and hole are $\hbar \omega_{c i}=\frac{\hbar l e \mid H}{m_{i} c} ; i=e, h$. The Schrödinger equation describing the Landau quantization for electron looks as

$$
\frac{1}{2} \hbar \omega_{c e}\left(\eta_{e}^{2}-\frac{\partial^{2}}{\partial \eta_{e}^{2}}\right)\left|\begin{array}{l}
\Phi_{1}\left(\eta_{e}\right) \\
\Phi_{2}\left(\eta_{e}\right)
\end{array}\right|-\frac{\alpha_{e} E_{z}}{l}\left(i \hat{\sigma}_{x} \frac{\partial}{\partial \eta_{e}}-\hat{\sigma}_{y} \eta_{e}\right)\left|\begin{array}{l}
\Phi_{1}\left(\eta_{e}\right) \\
\Phi_{2}\left(\eta_{e}\right)
\end{array}\right|=\varepsilon_{e}\left|\begin{array}{l}
\Phi_{1}\left(\eta_{e}\right) \\
\Phi_{2}\left(\eta_{e}\right)
\end{array}\right| .
$$

Introducing the dimensionless energy and SOC constant $\alpha$

$$
\varepsilon_{e}=\frac{\varepsilon_{e}}{\hbar \omega_{c e}} ; \alpha=\frac{\alpha_{e} E_{z}}{l \hbar \omega_{c e}}
$$

we can transcribe the two-component equation (14) as follows

$$
\begin{aligned}
& \frac{1}{2}\left(\eta_{e}^{2}-\frac{\partial^{2}}{\partial \eta_{e}^{2}}\right) \Phi_{1}\left(\eta_{e}\right)-i \alpha\left(\eta_{e}+\frac{\partial}{\partial \eta_{e}}\right) \Phi_{2}\left(\eta_{e}\right)=\varepsilon_{e} \Phi_{1}\left(\eta_{e}\right) ; \\
& \frac{1}{2}\left(\eta_{e}^{2}-\frac{\partial^{2}}{\partial \eta_{e}^{2}}\right) \Phi_{2}\left(\eta_{e}\right)+i \alpha\left(\eta_{e}-\frac{\partial}{\partial \eta_{e}}\right) \Phi_{1}\left(\eta_{e}\right)=\varepsilon_{e} \Phi_{2}\left(\eta_{e}\right) .
\end{aligned}
$$

Acting in the same way we will obtain the one variable hole Hamiltonian

$$
H_{h}\left(\eta_{h}\right)=\frac{1}{2} \hbar \omega_{c h}\left(\eta_{h}^{2}-\frac{\partial^{2}}{\partial \eta_{h}^{2}}\right) \hat{I}
$$




$$
-\frac{\beta_{h} E_{z}}{l^{3}}\left\{i \hat{\sigma}_{x}\left(\frac{\partial^{3}}{\partial \eta_{h}^{3}}+3 \eta_{h}^{2} \frac{\partial}{\partial \eta_{h}}+3 \eta_{h}\right)+\hat{\sigma}_{y}\left(\eta_{h}^{3}+3 \eta_{h} \frac{\partial^{2}}{\partial \eta_{h}^{2}}+3 \frac{\partial}{\partial \eta_{h}}\right)\right\} .
$$

The notations $\varepsilon_{h}=\frac{\varepsilon_{h}}{\hbar \omega_{c h}}, \quad \beta=\frac{\beta_{h} E_{z}}{l^{3} \hbar \omega_{c h}}$ and the relation

$$
\left(\eta \pm \frac{\partial}{\partial \eta}\right)^{3}=\eta^{3} \pm \frac{\partial^{3}}{\partial \eta^{3}}+3\left(\eta \frac{\partial^{2}}{\partial \eta^{2}} \pm \eta^{2} \frac{\partial}{\partial \eta}\right)+3\left(\eta \pm \frac{\partial}{\partial \eta}\right)
$$

permits to simplify essentially the two component Schrödinger equation

$$
\begin{aligned}
& \frac{1}{2}\left(\eta_{h}^{2}-\frac{\partial^{2}}{\partial \eta_{h}^{2}}\right) f_{1}\left(\eta_{h}\right)+i \beta\left(\eta_{h}-\frac{\partial}{\partial \eta_{h}}\right)^{3} f_{2}\left(\eta_{h}\right)=\varepsilon_{h} f_{1}\left(\eta_{h}\right) ; \\
& \frac{1}{2}\left(\eta_{h}^{2}-\frac{\partial^{2}}{\partial \eta_{h}^{2}}\right) f_{2}\left(\eta_{h}\right)-i \beta\left(\eta_{h}+\frac{\partial}{\partial \eta_{h}}\right)^{3} f_{1}\left(\eta_{h}\right)=\varepsilon_{h} f_{2}\left(\eta_{h}\right) .
\end{aligned}
$$

Rashba ${ }^{1}$ proposed the solution of equations (16) using the series expansions for the functions $\Phi_{1}$ and $\Phi_{2}$. We will use the same representations as follows

$$
\begin{gathered}
\Phi_{1}(\eta)=\sum_{n=0}^{\infty} a_{n} \varphi_{n}(\eta) ; \Phi_{2}(\eta)=\sum_{n=0}^{\infty} b_{n} \varphi_{n}(\eta) ; \\
f_{1}(\eta)=\sum_{n=0}^{\infty} c_{n} \varphi_{n}(\eta) ; f_{2}(\eta)=\sum_{n=0}^{\infty} d_{n} \varphi_{n}(\eta),
\end{gathered}
$$

where $\varphi_{n}(\eta)$ are the eigenfunctions of the Landau quantization in Landau gauge with the orthogonality and normalization conditions

$$
l \int_{-\infty}^{\infty} d \eta \varphi_{n}^{*}(\eta) \varphi_{m}(\eta)=\delta_{n m} ; \sum_{n}\left|a_{n}\right|^{2}+\sum_{n}\left|n_{n}\right|^{2}=1 ; \sum_{n}\left|c_{n}\right|^{2}+\sum_{n}\left|d_{n}\right|^{2}=1 .
$$

They obey to the differential equations

$$
\begin{gathered}
\left(\eta^{2}-\frac{\partial^{2}}{\partial \eta^{2}}\right) \varphi_{n}(\eta)=(2 n+1) \varphi_{n}(\eta), \\
\frac{1}{\sqrt{2}}\left(\eta-\frac{\partial}{\partial \eta}\right) \varphi_{n}(\eta)=\sqrt{(n+1)} \varphi_{n+1}(\eta), \\
\frac{1}{\sqrt{2}}\left(\eta+\frac{\partial}{\partial \eta}\right) \varphi_{n}(\eta)=\sqrt{n} \varphi_{n-1}(\eta), \\
\left(\eta-\frac{\partial}{\partial \eta}\right)^{3} \varphi_{n}(\eta)=2 \sqrt{2} \sqrt{(n+1)(n+2)(n+3)} \varphi_{n+3}(\eta), \\
\left(\eta+\frac{\partial}{\partial \eta}\right)^{3} \varphi_{n}(\eta)=2 \sqrt{2} \sqrt{n(n-1)(n-2)} \varphi_{n-3}(\eta) .
\end{gathered}
$$

As one can see the expressions $\frac{1}{\sqrt{2}}\left(\eta-\frac{\partial}{\partial \eta}\right)$ and $\frac{1}{\sqrt{2}}\left(\eta+\frac{\partial}{\partial \eta}\right)$ play the role of increasing and decreasing differential operators. The equalities (22) transform the Schrödinger equations (16) and (19) into the linear relations between the Landau quantization functions. They are

$$
\begin{gathered}
\sum_{n=0}^{\infty} a_{n}\left(n+\frac{1}{2}-\varepsilon_{e}\right) \varphi_{n}(\eta)-i \alpha \sum_{n=0}^{\infty} b_{n} \sqrt{2} \sqrt{n} \varphi_{n-1}(\eta)=0 \\
\sum_{n=0}^{\infty} b_{n}\left(n+\frac{1}{2}-\varepsilon_{e}\right) \varphi_{n}(\eta)+i \alpha \sum_{n=0}^{\infty} a_{n} \sqrt{2} \sqrt{n+1} \varphi_{n+1}(\eta)=0
\end{gathered}
$$

for $2 \mathrm{D}$ conduction electron, and

$$
\sum_{n=0}^{\infty} c_{n}\left(n+\frac{1}{2}-\varepsilon_{h}\right) \varphi_{n}(\eta)+i \beta 2 \sqrt{2} \sum_{n=0}^{\infty} d_{n} \sqrt{(n+1)(n+2)(n+3)} \varphi_{n+3}(\eta)=0
$$




$$
\sum_{n=0}^{\infty} d_{n}\left(n+\frac{1}{2}-\varepsilon_{h}\right) \varphi_{n}(\eta)-i \beta 2 \sqrt{2} \sum_{n=0}^{\infty} c_{n} \sqrt{n(n-1)(n-2)} \varphi_{n-3}(\eta)=0
$$

for 2D heavy holes.

Multiplying these equations by $\varphi_{s}^{*}(\eta)$, where $s=0,1,2, \ldots$, after the integration on the variable $\eta$ in accordance with the condition (21), we will obtain the linear algebraic equations.

In the case of the $2 \mathrm{D}$ heavy holes the algebraic linear equations are

$$
\begin{gathered}
c_{0}\left(\frac{1}{2}-\varepsilon_{h}\right)=0 ; c_{1}\left(\frac{3}{2}-\varepsilon_{h}\right)=0 ; c_{2}\left(\frac{5}{2}-\varepsilon_{h}\right)=0 \\
c_{3}\left(\frac{7}{2}-\varepsilon_{h}\right)=-i \beta 4 \sqrt{3} d_{0} ; d_{0}\left(\frac{1}{2}-\varepsilon_{h}\right)=i \beta 4 \sqrt{3} c_{3} \\
c_{4}\left(\frac{9}{2}-\varepsilon_{h}\right)=-i \beta 8 \sqrt{3} d_{1} ; d_{1}\left(\frac{3}{2}-\varepsilon_{h}\right)=i \beta 8 \sqrt{3} c_{4} ; \\
c_{5}\left(\frac{11}{2}-\varepsilon_{h}\right)=-i \beta 4 \sqrt{30} d_{2} ; d_{2}\left(\frac{5}{2}-\varepsilon_{h}\right)=i \beta 4 \sqrt{30} c_{5} ; \\
c_{6}\left(\frac{13}{2}-\varepsilon_{h}\right)=-i \beta 8 \sqrt{15} d_{3} ; d_{3}\left(\frac{7}{2}-\varepsilon_{h}\right)=i \beta 8 \sqrt{15} c_{6}, \ldots
\end{gathered}
$$

and so on. As in the case of conduction electron the solution $\varepsilon_{h}=\frac{1}{2}$ is accompanied by the coefficients $c_{0}=1$ and by all another coefficients $c_{n}$ and $d_{n}$ equal to zero. The second spin splitted lowest Landau level for a heavy hole has a value $\varepsilon_{h} \neq \frac{1}{2}$, what leads to the solutions $c_{0}=c_{1}=c_{2}=0$. The fourth and the fifth equations lead to the dispersion equation

$$
\left(\frac{7}{2}-\varepsilon_{h}\right)\left(\frac{1}{2}-\varepsilon_{h}\right)-48 \beta^{2}=0 ; \varepsilon_{h}=2 \pm \sqrt{\frac{9}{4}+48 \beta^{2}} ; c_{3}=\frac{-i \beta 4 \sqrt{3} d_{0}}{\frac{3}{2}+\sqrt{\frac{9}{4}+48 \beta^{2}}} .
$$

All coefficients except $d_{0}$ and $c_{3}$ are equal to zero, what leads to the equalities

$$
\left|c_{3}\right|^{2}+\left|d_{0}\right|^{2}=1 ;\left|d_{0}\right|^{2}=\frac{1}{1+\frac{48 \beta^{2}}{\left(\frac{3}{2}+\sqrt{\frac{9}{4}+48 \beta^{2}}\right)^{2}}} .
$$

In the limiting case $\beta^{2}<\frac{1}{64}$ the second solution is

$$
\varepsilon_{h} \square \frac{1}{2}-16 \beta^{2} ;\left|d_{0}\right|^{2} \square 1-\frac{16}{3} \beta^{2} ;\left|c_{3}\right|^{2} \square \frac{16}{3} \beta^{2} .
$$

Two spin splitted LLLs for hole are

$$
\begin{gathered}
\left|\Psi_{h}\left(R_{3}, q ; x, y\right)\right\rangle=\frac{e^{i q x}}{\sqrt{L_{x}}}\left|\begin{array}{c}
c_{3} \varphi_{3}(\eta) \\
d_{0} \varphi_{0}(\eta)
\end{array}\right| ; \varepsilon_{h}=2-\sqrt{\frac{9}{4}+48 \beta^{2}} \\
\left|\Psi_{h}\left(R_{4}, q ; x, y\right)\right\rangle=\frac{e^{i q x}}{\sqrt{L_{x}}}\left|\begin{array}{c}
\varphi_{0}(\eta) \\
0
\end{array}\right| ; \varepsilon_{h}=\frac{1}{2}
\end{gathered}
$$

In difference on the electron SOC parameter $\alpha=\frac{\alpha_{e} E_{z}}{l \hbar \omega_{c e}}$, which decreases with increasing magnetic field strength $H$, the hole SOC parameter $\beta=\frac{\beta_{h} E_{z}}{l^{3} \hbar \omega_{c h}}$ has an inverse dependence, i.e. it increases with the increasing $H$. Only at small values of $E_{z}$ and at not so high values of $H$ the parameter $\beta$ can be considered to be small, i.e. $\beta^{2}<\frac{1}{64}$.

There are four combinations of the electron and hole energies in the frame of these levels. They are represented in the Fig. 1 and are enumerated below: 


$$
\begin{gathered}
f_{1}=\left(e, R_{1} ; h, R_{3}\right) ; E\left(f_{1}\right)=E_{g}+\left(\frac{1}{2}-2 \alpha^{2}\right) \hbar \omega_{c e}+\left(\frac{1}{2}-16 \beta^{2}\right) \hbar \omega_{c h} \\
f_{2}=\left(e, R_{1} ; h, R_{4}\right) ; E\left(f_{2}\right)=E_{g}+\left(\frac{1}{2}-2 \alpha^{2}\right) \hbar \omega_{c e}+\frac{1}{2} \hbar \omega_{c h} \\
f_{3}=\left(e, R_{2} ; h, R_{3}\right) ; E\left(f_{3}\right)=E_{g}+\frac{1}{2} \hbar \omega_{c e}+\left(\frac{1}{2}-16 \beta^{2}\right) \hbar \omega_{c h} \\
f_{4}=\left(e, R_{2} ; h, R_{4}\right) ; E\left(f_{4}\right)=E_{g}+\frac{1}{2} \hbar \omega_{c e}+\frac{1}{2} \hbar \omega_{c h}
\end{gathered}
$$

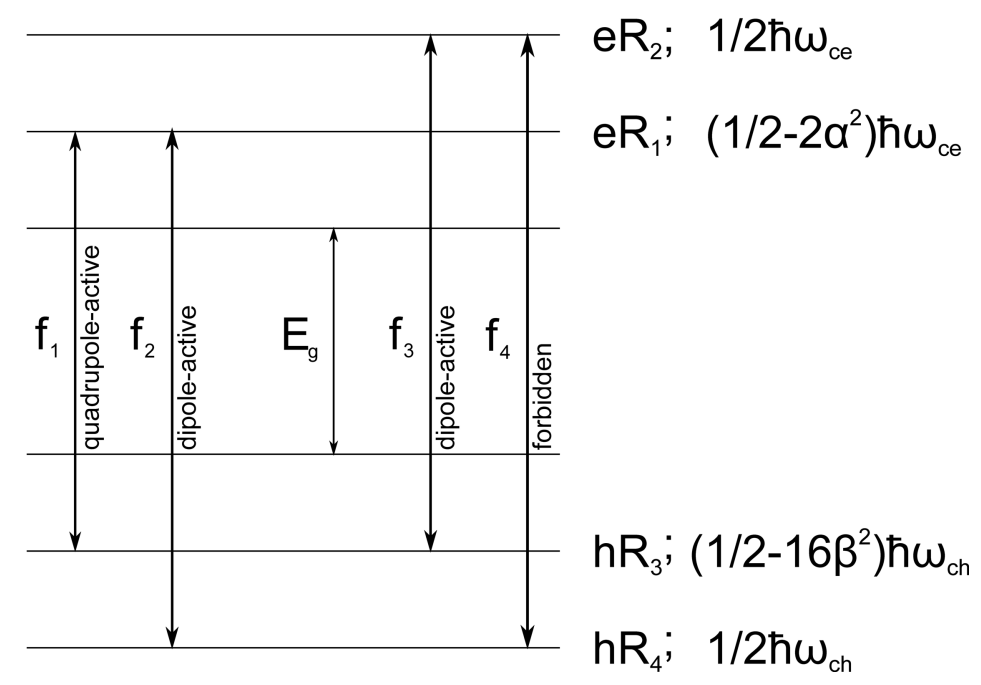

Fig. 1. Energy spectrum of the LLLs for electron and hole taking into account the RSOC. Four different combinations of the electronhole pair states are represented.

In the next section the matrix elements of the Coulomb e-h interaction will be calculated.

\section{THE COULOMB ELECTRON-HOLE INTERACTION, THE ENERGY SPECTRUM OF 2D MAGNETOEXCITONS AND THE BAND-TO-BAND QUANTUM TRANSITIONS}

The exciton wave functions in the $f_{s}$ compositions represented on the Fig. 1 are denoted as

$$
\Psi_{e x}\left(\vec{k}, f_{s}\right)=\frac{1}{\sqrt{N}} \sum_{t} e^{-i k_{y}, t^{2}} a_{R_{i}, \frac{k_{x}}{2}+t}^{\dagger} b_{R_{j}, \frac{k_{x}}{2} t}^{\dagger}|0\rangle ; \quad s=1,2,3,4 ; i=1,2 ; j=3,4,
$$

where $a^{\dagger}$ and $b^{\dagger}$ are the creation electron and hole operators.

The average values of the electron-hole Coulomb interaction Hamiltonian $H_{\text {Coul }}^{e-h}$ equals to

$$
\left\langle\Psi_{e x}\left(\vec{k}, f_{s}\right)\left|H_{\text {Coul }}^{e-h}\right| \Psi_{e x}\left(\vec{k}, f_{s}\right)\right\rangle=-I_{e x}\left(e, R_{i} ; h, R_{j} ; \vec{k}\right) ; \quad i=1,2 ; j=3,4 .
$$

The obtained values are represented on the figure 2 . 


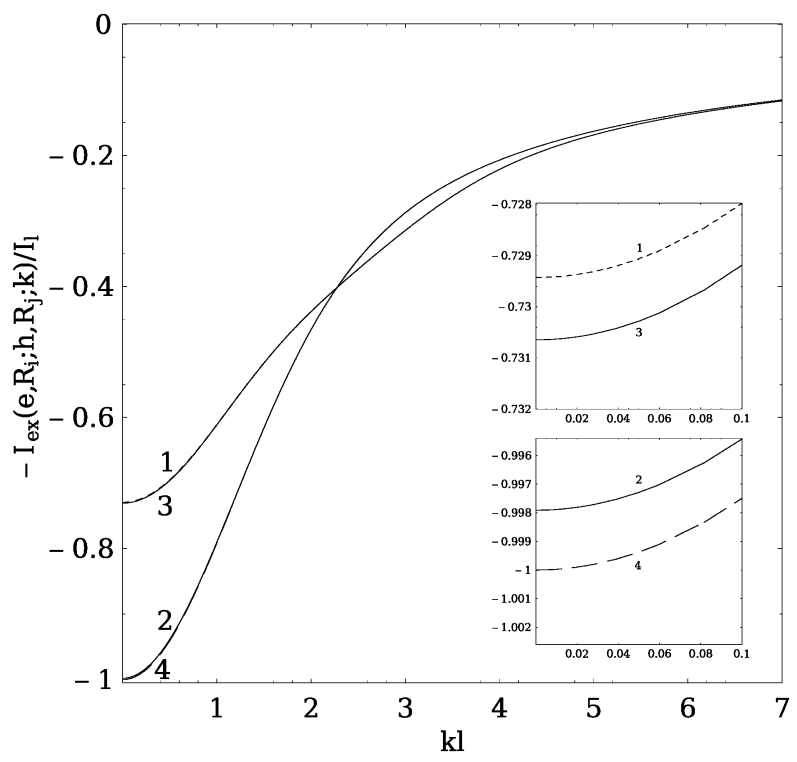

Fig. 2. The ionization potentials $I_{e x}\left(e, R_{i} ; h, R_{j} ; k\right) / I_{l}, i=1,2 ; j=3,4$ taken with the sign minus for spin-splitted LLLs for electrons and holes at $E_{z}=24 \mathrm{kV} / \mathrm{cm}$ and $H=15 \mathrm{~T}$. The insets demonstrate the datailed dependences of these magnetoexciton states 1) $\left.\left.\left.\left(e, R_{1} ; h, R_{3}\right) ; 2\right)\left(e, R_{1} ; h, R_{4}\right) ; 3\right)\left(e, R_{2} ; h, R_{3}\right) ; 4\right)\left(e, R_{2} ; h, R_{4}\right)$.

The probability of the quantum transition in the exciton state $f_{1}$ is different from zero only if the small corrections proportional to $\left(Q_{x}+i Q_{y}\right) l$ are taken into account. It means that the transition to the exciton state $f_{1}$ is proportional to $\left(Q_{x}^{2}+Q_{y}^{2}\right) l^{2}=\left|\vec{Q}_{2 D}\right|^{2} l^{2}$ and the corresponding quantum transition is quadrupole-active, being proportional to the square of the projection $\vec{Q}_{2 D}$ on the layer surface of the light wave vector $\vec{Q}$ arbitrary oriented in the 3D space. In the Faraday geometry, when the wave vector $\vec{Q}$ is parallel to the direction of the external perpendicular magnetic field, the projection $\vec{Q}_{2 D}=0$ and the quadrupole transition is forbidden. The quantum transitions in the states $f_{2}$ and $f_{3}$ are dipole active.

\section{CONCLUSIONS}

The influence of the Rashba spin-orbit coupling (RSOC) on the properties of the 2D magnetoexcitons was determined. The interdependence between the Landau quantization of the electron and hole orbital motions and their spin projections was revealed in the frame of Landau gauge. The spinor-type wave functions of the 2D conduction and valence electrons in the presence of the RSOC have different numbers of the Landau quantization functions for different spin projections. For example, they are $\varphi_{0}(y)$ and $\varphi_{1}(y)$ in one case, and $\varphi_{3}(y)$ and $\varphi_{0}(y)$ in another one. For conduction electron, if the number of Landau level is $n$ for the up spin projection, it is equal to $n+1$ for the down spin projection. For the valence electron and for the heavy hole $(\mathrm{HH})$ the number $n$ of the Landau level for the down spin projection is accompanied by the number $n+3$ for the up spin projection. It is determined completely by the fact that the RSOC Hamiltonian for conduction electron is linear in the projections $k_{ \pm}=k_{x} \pm i k_{y}$ of the in-plane wave vector $\vec{k}_{\|}$, whereas in the case of valent electron and heavy hole the corresponding Hamiltonian contains the third order of these projections, i.e. the expressions $\left(k_{ \pm}\right)^{3}$. Two lowest Landau levels $R_{1}$ and $R_{2}$ for conduction electron and two lowest hole states $R_{3}$ and $R_{4}$ were considered. The wave functions were used to calculate the matrix elements of the Coulomb direct and exchange electronhole (e-h) interactions corresponding to the combinations $f_{1}=\left(e, R_{1} ; h, R_{3}\right) ; f_{2}=\left(e, R_{1} ; h, R_{4}\right) ; f_{3}=\left(e, R_{2} ; h, R_{3}\right)$ and $f_{4}=\left(e, R_{2} ; h, R_{4}\right)$. The corresponding ionization potentials were expressed through the ionization potentials of the bare magnetoexciton states $I_{e x}^{(n, m)}(k)$ calculated earlier $i^{10}$. With their help as well as with the knowledge of the coefficients 
$d_{0}, c_{3}$ (28) it is possible to determine the dispersion laws (32) of the four new magnetoexciton bands taking into account the RSOC. The new dispersion laws could lead to new collective properties of the spinor-type 2D magnetoexcitons.

The optical quantum transitions from the ground state of the crystal to four magnetoexciton states were determined on the base of exciton wave functions (31) and electron-radiation interaction. It was shown that the quantum transitions in the states $f_{2}$ and $f_{3}$ corresponding to combinations $f_{2}=\left(e, R_{1} ; h, R_{4}\right)$ and $f_{3}=\left(e, R_{2} ; h, R_{3}\right)$ are dipole-active, the exciton state $f_{1}=\left(e, R_{1} ; h, R_{3}\right)$ is quadrupole-active, whereas the fourth combination $f_{4}=\left(e, R_{2} ; h, R_{4}\right)$ is forbidden. In the Faraday geometry, when the light wave vector $\vec{Q}$ is oriented along the magnetic field direction, the circular polarizations $\vec{\sigma}_{\bar{Q}}^{ \pm}$coincide with the exciton circular polarization $\vec{\sigma}_{ \pm 1}$. The light circular polarization $\vec{\sigma}_{\bar{Q}}^{\mp}$ excites the exciton states $\vec{\sigma}_{\mp 1}$ because $\left(\vec{\sigma}_{\vec{Q}}^{\mp *} \cdot \vec{\sigma}_{\mp 1}\right)=\left(\vec{\sigma}_{\bar{Q}}^{ \pm} \cdot \vec{\sigma}_{\mp 1}\right)=1$, whereas $\left(\vec{\sigma}_{\vec{Q}}^{ \pm *} \cdot \vec{\sigma}_{\mp 1}\right)=0$. Such optical orientation of the exciton states under the influence of the circularly polarized light is named as optical alignment ${ }^{13}$. In $\vec{\sigma}_{\bar{Q}}$ polarization only the dipole-active quantum transition $f_{2}$ is allowed, whereas the dipole-active quantum transition $f_{3}$ and the quadrupoleactive quantum transition $f_{1}$ are forbidden. In Faraday geometry and circular polarization $\vec{\sigma}_{\bar{Q}}^{+}$the exciton states $f_{1}$ and $f_{3}$ having the circular polarization $\vec{\sigma}_{1}$ are allowed. One is dipole-active and another one is quadrupole-active.

\section{REFERENCES}

[1] Rashba, E.I., Sov. Phys. Fiz. Tverd. Tela (Leningrad) 2, 1224 (1960).

[2] Hakioğlu, T. and Şahin, M., Phys. Rev. Lett. 98, 166405 (2007).

[3] Ali Can, M., and Hakioğlu, T., Phys. Rev. Lett. 103, 086404 (2009).

[4] Dresselhaus, G., Phys. Rev. 100, 580 (1955).

[5] Bychkov, Yu.A. and Rashba, E.I., Sov. Phys. Pis'ma JETP 39, 66 (1984).

[6] Winkler, R., Phys. Rev. B 62, 4245 (2000).

[7] Winkler, R., Noh, H., Tutuc, E., and Shayegan, M., Phys. Rev. B 65, 155303 (2002).

[8] Bir, G.L. and Pikus, G.E., [Symmetry and Strain-induced Effects in Semiconductors], Wiley, New York, (1974).

[9] Moskalenko, S.A., Snoke, D.W., [Bose-Einstein Condensation of Excitons and Biexcitons and Coherent Nonlinear Optics with Excitons], Cambridge University Press, Cambridge, 415 pages (2000).

[10] S.A. Moskalenko, M.A. Liberman, P.I. Khadzhi, E.V. Dumanov, Ig.V. Podlesny, and V.V. Boțan, Physica E 39, 137 (2007).

[11] Moskalenko, S.A., [Introduction in the theory of high density excitons], Chisinau, Shtiintsa, (1983), in Russian.

[12] Moskalenko, S.A., Liberman, M.A., Podlesny, I.V., Phys. Rev. B 79, 125425 (2009).

[13] Series editors Agranovich, V.M., Maradudin, A.A., [Optical orientation], volume editors Meier, F., and Zakharchenya, B.P., Vol. 8. [Modern problems in condensed matter sciences], North-Holland, Amsterdam, Oxford, New-York, Tokyo, (1984). 